\title{
Evaluación electrofisiológica del procesamiento auditivo
}

\section{Electrophysiological assessment of auditory processing}

\author{
Anthony Marcotti F. ${ }^{1,2}$, Sebastián Rivera $R^{3}$
}

${ }^{1}$ Carrera de Fonoaudiología,

Departamento de Ciencias

de la Salud, Facultad

de Medicina, Pontificia

Universidad Católica de Chile. Santiago, Chile

${ }^{2}$ Escuela de Fonoaudiología,

Facultad de Ciencias de la

Salud, Universidad de las

Américas. Santiago, Chile.

${ }^{3}$ Escuela de Fonoaudiología,

Facultad de Salud, Universidad

Santo Tomás. Viña del Mar

Chile.

Los autores declaran no tener conflictos de interés.

Recibido el 14 de octubre de 2019. Aceptado el 2 de septiembre de 2020

Correspondencia: Anthony Marcotti $\mathrm{F}$.

Departamento de Ciencias de la Salud, Pontificia Universidad Católica de Chile.

Av. Vicuña Mackenna 4860

Macul. Santiago, Chile.

Email: anthony.marcotti@uc.c

\section{Resumen}

En la actualidad existen distintas herramientas para la evaluación del procesamiento auditivo y sus alteraciones. Además de las pruebas conductuales y de las mediciones electroacústicas, se ha establecido que la batería mínima de evaluación del procesamiento auditivo debe incluir exámenes electrofisiológicos que permitan dar cuenta del estado funcional de las estructuras anatómicas a nivel de tronco cerebral, la corteza auditiva y del sistema nervioso auditivo central en general. Las pruebas electrofisiológicas más descritas en la literatura corresponden a las respuestas auditivas complejas de tronco cerebral, potenciales evocados auditivos de latencia media, potenciales evocados auditivos de latencia tardía, y los potenciales auditivos asociados a eventos como el potencial de disparidad y el P300. A pesar de que diversos organismos internacionales como la American Speech-Language-Hearing Association y la American Academy of Audiology han recomendado su utilización en el proceso diagnóstico del trastorno del procesamiento auditivo y han mencionado su utilidad en la diferenciación de cuadros asociados, no existe evidencia concluyente en relación con sus aplicaciones clínicas. Esto último ha llevado a organizaciones como la British Society of Audiology a poner en duda su verdadera utilidad en estos casos.

Palabras clave: procesamiento auditivo, trastorno del procesamiento auditivo, electrofisiología auditiva.

\begin{abstract}
Currently, there are different tools for the assessment of auditory processing and its disorders. In addition to behavioral tests and electroacoustic measurements, it has been established that the minimum battery for auditory processing assessment must include electrophysiological examinations that allow to verify the functional state of anatomical structures at the brainstem, the auditory cortex and, broadly, the central auditory nervous system. In literature, the most described electrophysiological tests correspond to complex auditory brainstem responses, auditory middle latency evoked responses, auditory late latency evoked responses and event related evoked potentials, such as mismatch negativity and P300. Although several international organizations such as the American Speech-Language-Hearing Association and the American Academy of Audiology have recommended its use in the diagnostic process of auditory processing disorder and have mentioned its usefulness in the differentiation of associated conditions, there is no conclusive evidence in relation to its clinical applications. The latter has led organizations such as the British Society of Audiology to question its true usefulness in these cases.
\end{abstract}

Keywords: auditory processing, auditory processing disorder, auditory electrophysiology.

\section{Introducción}

El trastorno del procesamiento auditivo (TPA) se refiere a una alteración en el procesamiento perceptual de la información acústica en el sistema nervioso auditivo central (SNAC), evidenciada en dificultades en las habilidades auditivas de localización y lateralización sonora, discriminación auditiva, reconocimiento de patrones auditivos, aspectos temporales de la audición, desempeño con señales acústicas competitivas y desempeño con señales acústicas degradadas ${ }^{1}$. Estas dificultades se caracterizan por producir una mala 
percepción tanto de sonidos verbales como no verbales, así como también por tener su origen en una función neural deteriorada ${ }^{2}$. Este trastorno puede involucrar a las vías aferentes y eferentes del mismo SNAC, así como a otros sistemas de procesamiento neural que le proporcionan la modulación top-down (de arriba hacia abajo), incluidos la visión y funciones cognitivas como el lenguaje, la atención, la función ejecutiva, el razonamiento, la memoria e incluso las emociones ${ }^{2}$.

En el año 2000, se realizó una conferencia en Estados Unidos cuya finalidad fue establecer lineamientos en la evaluación del procesamiento auditivo en niños de edad escolar ${ }^{3}$. Los participantes establecieron que la batería mínima de evaluación del procesamiento auditivo debe incluir, junto con las pruebas psicoacústicas y las mediciones electroacústicas, exámenes electrofisiológicos que permitan dar cuenta del estado de las estructuras anatómicas a nivel de tronco cerebral y corteza auditiva. En una declaración de consenso de la American Speech-Language-Hearing Association (ASHA), se sugiere además que este tipo de evaluación puede ser particularmente útil en los casos en que los procedimientos conductuales no son factibles, cuando existe la sospecha de un trastorno neurológico de base, cuando se necesita una confirmación de los hallazgos conductuales o cuando los hallazgos conductuales no son concluyentes ${ }^{1}$.

La American Academy of Audiology (AAA) en su documento sobre lineamientos sobre diagnóstico y tratamiento del TPA en niños y adultos, describe las técnicas electrofisiológicas como herramientas de gran valor en el proceso de evaluación del procesamiento auditivo ${ }^{4}$. En el documento, se menciona la utilidad de obtener información desde el tronco cerebral hasta la corteza auditiva a través de los potenciales evocados de tronco cerebral, respuestas auditivas de latencia media, respuestas auditivas de latencia tardía y potenciales evocados relacionados a eventos, como el P300 y el potencial de disparidad. La AAA propone un análisis cauteloso de los resultados electrofisiológicos ya que, a pesar de que pueden proporcionar información objetiva con respecto a la transmisión de señales acústicas a través del SNAC, existen casos en que las respuestas neurofisiológicas pueden ser completamente normales. Detalla que existen muchos casos de TPA en los cuales la electrofisiología no es capaz de demostrar alguna alteración debido a lo heterogéneas de sus formas de presentación.

A pesar de las recomendaciones de la ASHA y la AAA, no existen criterios bien establecidos sobre cuándo debe incluirse la electrofisiología en la evaluación clínica del TPA. Para la British Society of Audiology (BSA), la evidencia disponible en la actualidad no es suficiente para justificar la inclusión de estas pruebas en casos de audiometría normal, con la excepción del potencial evocado auditivo de tronco cerebral (PEATC) que, cuando se usa con emisiones otoacústicas y/o potenciales microfónicos cocleares, facilitaría la identificación de los trastornos del espectro de la neuropatía auditiva ${ }^{2}$. Si bien se reconoce la utilidad de estas medidas para brindar información sobre el SNAC de manera objetiva, la confiabilidad y, por ende, la utilidad clínica de estas técnicas de evaluación se ven limitadas por la diversidad de estímulos, técnicas y protocolos descritos en la literatura. A esto se suma la existencia de otras limitaciones como, la ausencia de valores normativos, gran variabilidad intersujeto, gran dificultad para su obtención a través de equipamiento clínico, gran variabilidad asociada a la edad y en algunos casos, dependencia de factores cognitivos. Estos alcances abarcan una gran variedad de técnicas, como los potenciales de tronco con estímulos complejos, respuestas de latencia media, respuestas corticales e incluso respuestas relacionadas a eventos ${ }^{5}$.

El objetivo de la presente revisión es describir las experiencias reportadas en la literatura en relación con la evaluación del procesamiento auditivo a través de técnicas electrofisiológicas. Por ser las más utilizadas y referenciadas en la literatura, el presente documento se centró en las respuestas auditivas complejas de tronco cerebral, potenciales evocados auditivos de latencia media, potenciales evocados auditivos de latencia tardía, y los potenciales auditivos asociados a eventos como el potencial de disparidad y el P300. Dentro de la revisión se han incorporado algunas experiencias relacionadas con cuadros clásicamente asociados al TPA, como problemas de aprendizaje, trastornos específicos de lenguaje y lesiones en el SNAC debido a que son condiciones que presentan una alta comorbilidad ${ }^{1}$. Además, en muchos 
lugares, la evaluación del procesamiento auditivo no es un procedimiento habitual y, a pesar de que estas otras alteraciones no reciben una etiqueta diagnóstica formal de TPA, las dificultades en una o más habilidades auditivas estaría presente en la gran mayoría de los casos ${ }^{5}$.

\section{Respuestas auditivas complejas de tronco cerebral}

Las respuestas auditivas complejas de tronco cerebral o cABR (complex auditory brainstem response), son el reflejo de la sincronización neural y la codificación de las características temporales de los sonidos, principalmente del habla a nivel del tronco cerebral ${ }^{6}$. A diferencia de un ABR convencional y como el nombre lo indica, se utilizan estímulos complejos para evocar las respuestas, siendo utilizados principalmente sonidos de habla como vocales o sílabas, naturales, sintéticas o híbridas ${ }^{7}$, aunque también hay reportes de la utilización de sonidos no verbales como el llanto de un bebé ${ }^{8}$ y estímulos musicales ${ }^{9}$. Independiente de su naturaleza, los estímulos utilizados deben ser estructuras formadas por elementos armónicos con cambios espectrales y temporales rápidos que, por lo tanto, requieran una respuesta neural sincronizada para permitir una decodificación precisa ${ }^{10}$. Generalmente, se recomienda la utilización de estímulos verbales sintéticos ya que permiten la manipulación y modificación de algunos parámetros acústicos de la señal ${ }^{6}$, a diferencia de los estímulos grabados de manera natural. Esto facilitaría la obtención de resultados altamente reproducibles y con una menor variabilidad test-retest ${ }^{11}$.

Al menos a nivel de tronco cerebral, con los estímulos complejos se pueden evidenciar dos clases de respuestas sincronizadas en tiempo (time-locked): transitorias y sostenidas ${ }^{10}$. Como los mismos nombres lo sugieren, las características breves y no periódicas del estímulo evocan las respuestas transitorias, mientras que las características periódicas del estímulo evocan las respuestas sostenidas. Estas últimas, además, pueden estar sincronizadas en fase (phase-locked). Al utilizar la sílaba /da/ como estímulo verbal (uno de los estímulos más utilizados), se obtienen respuestas tanto transitorias como sostenidas, logrando identificar picos de respuesta característicos que han sido denominados $\mathrm{V}, \mathrm{Va}, \mathrm{C}, \mathrm{D}, \mathrm{E}, \mathrm{F} \mathrm{y} \mathrm{O}^{7}$ (Figura 1). El complejo V-Va corresponde a los primeros componentes transitorios, siendo análogos a

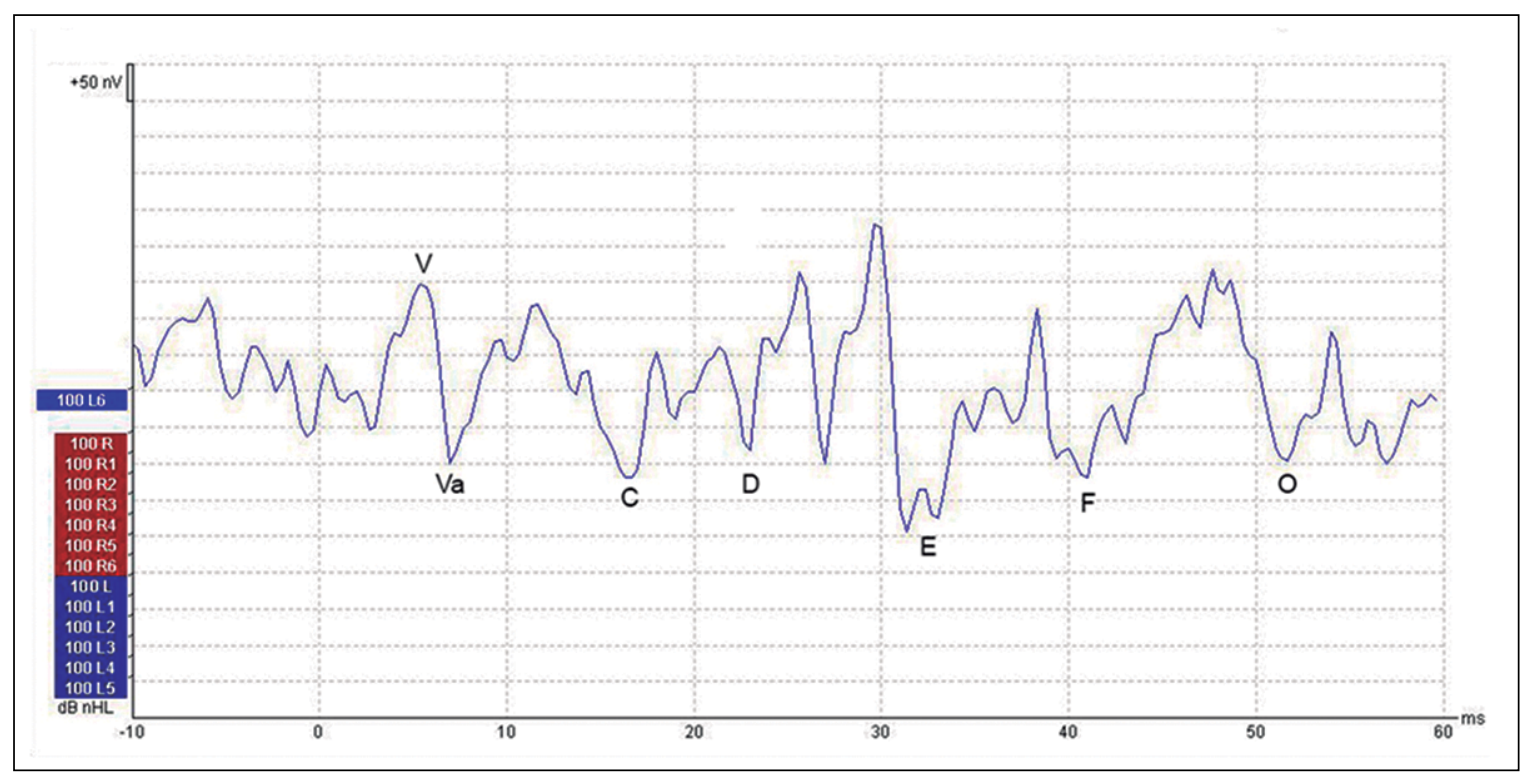

Figura 1. Respuesta auditiva compleja de tronco cerebral obtenido a través de un estímulo verbal sintético de sílaba /da/. Se identifican los componentes transitorios $\mathrm{V}, \mathrm{Va}, \mathrm{C}$ y $\mathrm{O}$, además de los componentes sostenidos $\mathrm{D}, \mathrm{E}$ y $\mathrm{F}$. 
la onda V obtenida a través de estimulación convencional. Los otros dos componentes transitorios son la onda $\mathrm{C}$ y $\mathrm{O}$, que corresponden respectivamente al comienzo y final (onset $y$ offset) de la sonoridad (vibración de los pliegues vocales) del estímulo. Los componentes D, E y F son respuestas sostenidas que forman la frequency following response (FFR), correspondiendo básicamente a la frecuencia fundamental (F0) del estímulo y a sus armónicos dentro de la transición formántica consonante-vocal.

La utilidad de los cABR se ha estudiado en una gran variedad de condiciones patológicas, tanto en niños como en adultos. En menores con trastornos del aprendizaje (TEA), se ha evidenciado que el complejo V-Va ha obtenido una pendiente significativamente menor, latencias aumentadas y/o amplitudes disminuidas en comparación a pares de la misma edad, pero sin dificultades de aprendizaje ${ }^{12}$. Este hallazgo sería común para niños con TPA, en los cuales también se ha documentado un aumento de latencia y disminuciones de amplitud para el complejo $\mathrm{V}$-Va, además de una amplitud disminuida para el componente $\mathrm{C}$ en comparación a sujetos sin quejas de procesamiento auditivo ${ }^{13}$. Otro componente que ha demostrado ser un elemento diferenciador entre menores con TEA es el FFR, el cual tendría una amplitud disminuida en relación con pares sin dificultades ${ }^{12}$. Estos antecedentes posicionan a los cABR como una potente herramienta para el estudio del procesamiento auditivo, trastornos de lenguaje y del aprendizaje en general, ya que, al menos el complejo $\mathrm{V}$-Va y el FFR, son independientes de las habilidades cognitivas y de la inteligencia del sujeto evaluado, por lo que entregarían información exclusivamente auditiva logrando, además, diferenciar sujetos con alteración de normales. Otras técnicas, como los ABR clásicos mediante estímulo clic o estímulos frecuenciales, a pesar de reflejar actividad subcortical y no dependen de habilidades cognitivas, no lograría retratar esta última diferenciación ${ }^{13}$. A pesar de sus posibles utilidades, no se han encontrado valores o reportes de sensibilidad y especificidad de los cABR para ningún tipo de condición patológica.

Los cABR también han demostrado ser útiles como marcador neurofisiológico para el monitoreo de las intervenciones formales para TPA. Filippini y colaboradore ${ }^{14}$ realizaron un estudio sobre los efectos de un programa de entrenamiento auditivo formal en 9 niños con TPA y 6 niños con trastornos específicos del lenguaje (TEL) en comparación a un grupo de 7 niños con desarrollo típico y otro grupo de 8 menores con TEL que no fueron sometidos a entrenamiento auditivo. Todos los menores tenían entre 7 y 13 años. Se realizaron mediciones de cABR evocados por un estímulo /da/ en silencio y en presencia de ruido ipsilateral con una relación señal ruido (SNR) de $+5 \mathrm{~dB}$ antes y después del entrenamiento. De los grupos que fueron sometidos a entrenamiento auditivo, el grupo con TPA demostró reducciones significativas en la latencia de los componentes $\mathrm{V}$ y A en condiciones de ruido, mientras que el grupo de menores con TEL mostró un acortamiento de las latencias de los componentes V, C, D y E en la misma condición. Los dos grupos que no recibieron entrenamiento no presentaron modificaciones en sus respuestas.

Otro antecedente para tener en consideración es la correlación de las mediciones de cABR con las pruebas conductuales de procesamiento auditivo. Un estudio realizado en un grupo de adultos con pérdida auditiva sensorioneural hasta de grado moderado ${ }^{15}$, no evidenció correlaciones significativas con las pruebas de detección de gaps aleatorios (RGDT) y de diferencias de nivel de enmascaramiento (MLD). Todas las mediciones de cABR estuvieron alteradas, presentando latencias aumentados o con ausencia de los componentes onset y/o FFR, y la mayoría de las respuestas en las pruebas conductuales se encontraron dentro de los rangos de normalidad. Sin embargo, pese a no encontrar alguna correlación significativa, los investigadores concluyeron que la evaluación electrofisiológica con estímulos verbales no depende únicamente del rendimiento de las habilidades conductuales y que, además, este técnica en particular permitiría detectar alteraciones más sutiles en el procesamiento auditivo producto de deprivaciones auditivas a nivel periférico, como las que se han reportado previamente en casos de hipoacusia de conducción ${ }^{16}$, hipoacusia sensorioneural unilatera ${ }^{17} \mathrm{y}$ bilateral ${ }^{18}$. Estos y los demás hallazgos se resumen en la Tabla 1. 
Tabla 1. Resumen de las principales investigaciones y sus hallazgos sobre la utilidad de los cABR en el estudio del procesamiento auditivo y sus alteraciones

\begin{tabular}{|c|c|c|c|c|c|c|}
\hline Estudio & Año & Alteración & $n$ & Edades & Descripción & Hallazgos \\
\hline $\begin{array}{l}\text { Wible y } \\
\text { cols. }^{12}\end{array}$ & 2004 & TEA & 11 & Niños & $\begin{array}{l}\text { Comparar con } \\
\text { sujetos normales }\end{array}$ & $\begin{array}{l}\text { - Componente V-Va con } \\
\text { pendiente y amplitud reducida, } \\
\text { latencia aumentada } \\
\text { - FFR de amplitud reducida }\end{array}$ \\
\hline $\begin{array}{l}\text { Filippini y } \\
\text { Schochat }^{13}\end{array}$ & 2009 & $\begin{array}{l}\text { TPA y quejas } \\
\text { afines }\end{array}$ & 20 & 7 a 24 & $\begin{array}{l}\text { Comparar con } \\
\text { sujetos normales }\end{array}$ & $\begin{array}{l}\text { - Componente V-Va con } \\
\text { pendiente y amplitud reducida, } \\
\text { latencia aumentada } \\
\text { - Componente C con amplitud } \\
\text { reducida }\end{array}$ \\
\hline $\begin{array}{l}\text { Filippini y } \\
\text { cols. }{ }^{14}\end{array}$ & 2012 & TPA y TEL & 23 & 7 a 13 & $\begin{array}{c}\text { Comparar con } \\
\text { sujetos normales } \\
\text { pre y } \\
\text { posentrenamiento } \\
\text { auditivo }\end{array}$ & $\begin{array}{l}\text { - Latencia de componente } \\
\text { V-Va y D aumentadas pre } \\
\text { entrenamiento y reducción de } \\
\text { estas en el posentrenamiento en } \\
\text { el grupo con TPA } \\
\text { - Grupo de TEL con reducción de } \\
\text { latencias de componentes V, C, } \\
\text { D y E posentrenamiento }\end{array}$ \\
\hline $\begin{array}{l}\text { Sanguebuche } \\
\text { y cols. }{ }^{15}\end{array}$ & 2018 & $\begin{array}{l}\text { HSN bilateral } \\
\text { moderada }\end{array}$ & 8 & 45 a 58 & $\begin{array}{c}\text { Correlacionar con } \\
\text { pruebas conductuales } \\
\text { RGDT y MLD }\end{array}$ & $\begin{array}{l}\text { - Latencia aumentada o ausencia } \\
\text { de los componentes O y/o FRR }\end{array}$ \\
\hline
\end{tabular}

TEA: trastorno específico de aprendizaje; TPA: trastorno del procesamiento auditivo; TEL: trastorno específico de lenguaje; HSN: hipoacusia sensorioneural; RGDT: prueba de detección de gaps aleatorios; MLD: prueba de diferencia de nivel de enmascaramiento.

\section{Potenciales evocados auditivos de latencia media (PEALM)}

Los potenciales evocados auditivos de latencia media, llamados también respuestas auditivas de latencia media o AMLR (auditory middle latency response), corresponden a un complejo electrofisiológico bifásico compuesto por cuatro ondas que aparecen entre los 10 a 80 primeros milisegundos tras una estimulación auditiva ${ }^{19}$ y que han obtenido clásicamente a través de estímulos clic, tonos burst y estímulos verbales de corta duración ${ }^{20}$. Se registraron por primera vez en humanos en el año 1958 en el Laboratorio de Investigación de Electrónica del Massachusetts Institute of Technology ${ }^{21}$. Se caracterizan por ser ondas de mayor amplitud y con una frecuencia fundamental más baja que las respuestas clásicas del ABR. Hasta antes de la masificación del cABR, estas respuestas habían sido consideradas como una de las técnicas objetivas de evaluación más prometedoras para el estudio de las alteraciones del SNAC y del TPA ${ }^{22}$. Sin embargo, se debe considerar que estas respuestas son consideradas mesógenas, es decir, se caracterizan por depender tanto de factores extrínsecos, por ejemplo, el tipo de estimulación o parámetros de registro, como de factores intrínsecos tales como nivel de vigilia $\mathrm{o}$ atención a los estímulos ${ }^{20}$.

Las etiquetas que se utilizan hoy en día para nombrar los componentes del AMLR fueron introducidas por primera vez en $1967^{23}$. Se decidió utilizar las letras "P" $\mathrm{y}$ " $\mathrm{N}$ " para designar a aquellos picos positivos y negativos respectivamente, pero para evitar confusiones con las ya utilizadas respuestas de latencia tardía (como P1, N1, P2 y N2), se decidió agregar una letra minúscula para diferenciar estas ondas según su orden de aparición. De esta manera, las ondas del AMLR quedaron designadas de la siguiente forma según su orden de ocurrencia: $\mathrm{Na}, \mathrm{Pa}, \mathrm{Nb}$ y $\mathrm{Pb}$ (Figura 2). Los centros generadores de estos componentes son ambiguos, 


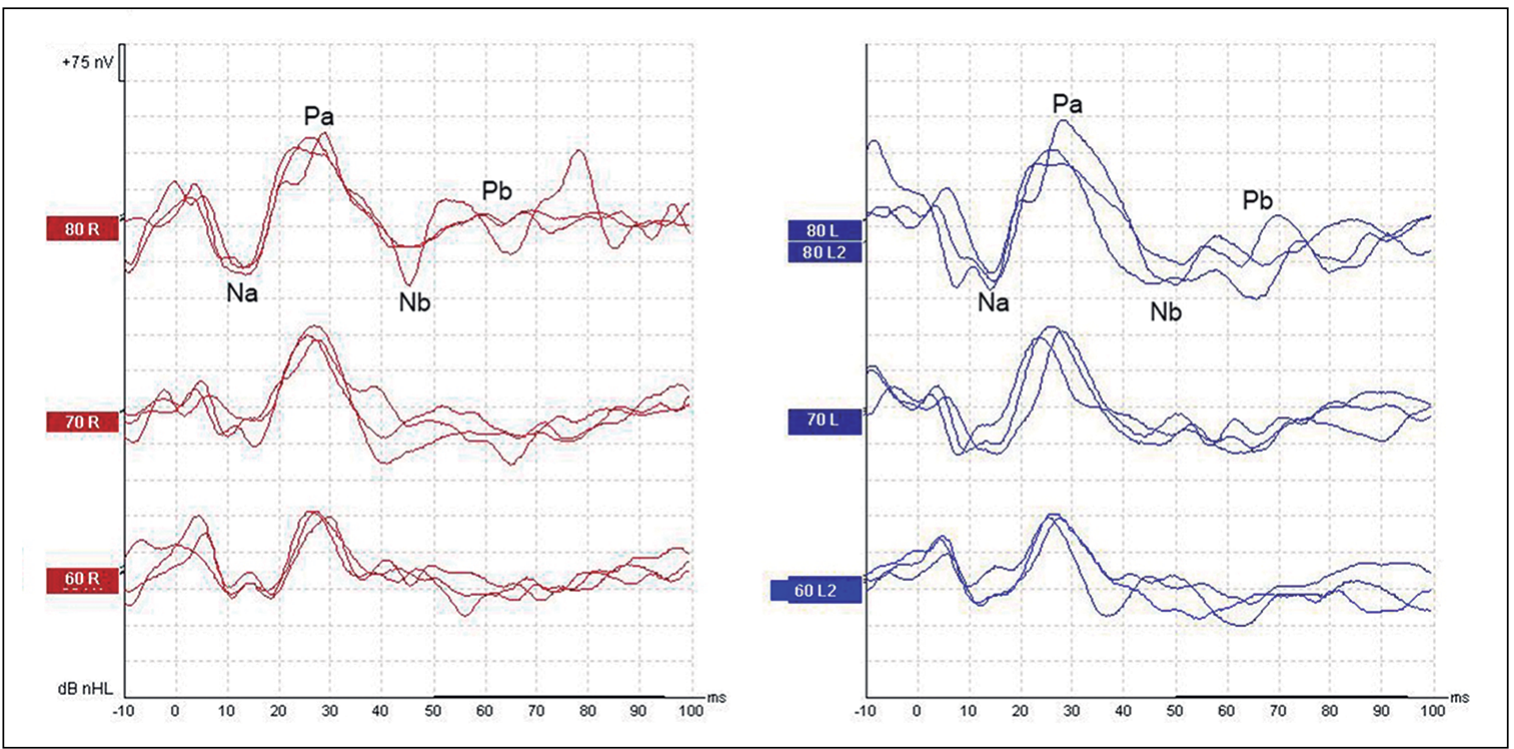

Figura 2. Potencial evocado auditivo de latencia media obtenido a través de un estímulo burst de $2000 \mathrm{~Hz}$. Se evidencian los componentes $\mathrm{Na}, \mathrm{Pa}, \mathrm{Nb}$ y $\mathrm{Pb}$.

atribuyendo su origen tanto a estructuras corticales como subcorticales. Se han evidenciado aportes del colículo inferior dentro de la región del mesencéfalo ${ }^{24}$, proyecciones talamocortica$\operatorname{les}^{25}$, del sulcus, porción posteromedial del giro de Heschl, plano temporal y del giro temporal superior $^{26}$. A diferencia de los componentes $\mathrm{Nb}$ y $\mathrm{Pb}$, los componentes $\mathrm{Na}$ y $\mathrm{Pa}$ no reciben prácticamente ninguna influencia de la formación reticular, lo que los haría que se mantuvieran más o menos estables independientes del estado de vigilia del sujeto ${ }^{27}$.

En un estudio en niños con $\mathrm{TEA}^{28}$, se evidenció que la latencia del componente $\mathrm{Na}$ fue significativamente mayor y que el componente $\mathrm{Nb}$ presentó una menor amplitud en relación con sujetos con desarrollo normal. En un estudio similar ${ }^{31}$, también se logró evidenciar que los menores con TEA tienen una mayor latencia del componente $\mathrm{Pa} \mathrm{y} \mathrm{Nb}$, principalmente en los registros obtenidos del hemisferio izquierdo. Si bien, los trastornos del aprendizaje constituirían una categoría diagnóstica distinta al TPA, este último sería la causa de algunas dificultades específicas en el aprendizaje o concomitaría habitualmente con ellas ${ }^{29}$. En niños con TPA, se han evidenciado alteraciones relacionadas con la amplitud de los componentes, principalmente en el componente $\mathrm{Na}-\mathrm{Pa}$, que ha presentado amplitudes reducidas en relación con sujetos con desarrollo normal ${ }^{30}$, aunque también se han reportado latencias aumentadas en el componente $\mathrm{Pa}$, principalmente en el registro obtenido del hemisferio izquierdo ${ }^{33}$. Junto con esto, y a diferencia de otras técnicas electrofisiológicas, se ha estudiado la sensibilidad/ especificidad de los AMLR para el diagnóstico de TPA. Se ha descrito que efecto el electrodo (diferencias de amplitud entre electrodos de distintos hemisferios) presenta una sensibilidad del $50 \%$ para TPA y de 55,6\% para lesiones del SNAC con una especificidad del $55,6 \%$, mientras que el efecto oído (diferencia de amplitud en uno o más electrodos según el oído estimulado) presenta una sensibilidad del $76,5 \%$ para TPA y $70 \%$ para lesiones del SNAC con una especificidad del $55,6 \%$ con un criterio de corte de asimetría del $30 \%{ }^{22}$. Estos valores fueron reportados estudiando la amplitud del componente Pa por tratarse de uno de los componentes más estables y robustos de los AMLR.

$\mathrm{Al}$ igual que los cABR, los AMLR también han demostrado ser útiles para monitorear y evaluar los resultados de intervenciones au- 
ditivas. En el año 2010, se realizó un estudio en 30 sujetos entre 8 y 14 años diagnosticados con TPA, los cuales fueron sometidos a entrenamiento auditivo formal e informal y sus resultados fueron comparados con un grupo control de 22 individuos con desarrollo típico que no fueron sometidos a ninguna intervención $^{30}$. El entrenamiento auditivo formal incluyó entrenamiento de percepción de frecuencia, intensidad, aspectos temporales, escucha dicótica, localización sonora y percepción de habla. El entrenamiento informal incluyó recontado de historias, identificación de elementos importantes de una narración, entre otros. El grupo estudio presentó diferencias significativas después del entrenamiento en las amplitudes del componente $\mathrm{Na}-\mathrm{Pa}$. No se encontraron diferencias significativas en relación con las latencias. Al realizar la comparación entre grupos, se evidenciaron diferencias significativas en las amplitudes antes del entrenamiento, sin embargo, después de esto, el grupo de menores con TPA mostró mayores amplitudes, similares a las del grupo control. Los autores concluyeron que la amplitud del complejo $\mathrm{Na}-\mathrm{Pa}$ no solo es un elemento diferenciador entre sujetos con desarrollo típico y con TPA, sino que además sería un marcador neurobiológico del éxito terapéutico en sujetos con TPA.

En cuanto a la correlación de los AMLR con pruebas conductuales de procesamiento auditivo, los hallazgos parecen indicar que existiría algún grado de concordancia entre ambos tipos de mediciones cuando se estiman los cambios producto de un entrenamiento auditivo, sea este formal o informal. En uno de los estudios mencionados anteriormente ${ }^{30}$, si bien no se realizó un estudio formal de la correlación, se evidenciaron mejoras significativas en las pruebas índice de habla pediátrico (PSI), habla en ruido (SIN), palabras espondaicas escalonadas (SSW) y dígitos dicóticos (DD) en una condición posintervención, lo cual coincidió con un incremento significativo en las amplitudes del componente Na-Pa. Resultados similares se evidenciaron en el estudio de Purdy y colaboradores ${ }^{28}$ en un grupo de niños con TEA, en los cuales se evidenciaron alteraciones en la latencia del componente $\mathrm{Na}$ y en la amplitud del componente $\mathrm{Nb}$, concordando con los resultados de la prueba SSW y con el reporte subjetivo de profesores. En este mismo estudio, sin embargo, no se evidenció algún nivel de concordancia con la prueba palabras competitivas $(\mathrm{CW})$. En relación con otro tipo de pruebas conductuales, se ha evidenciado una correlación significativa negativa entre habilidades de conciencia fonológica medida por el instrumento de evaluación secuencial CONFIAS $^{32}$ y la latencia de los componentes $\mathrm{Na}, \mathrm{Pa}$ y $\mathrm{Nb}$ y una correlación positiva con la amplitud del complejo $\mathrm{Na}-\mathrm{Pa}$. Estos resultados dejan en evidencia la utilidad del AMLR para identificar hallazgos auditivos específicos, además de alteraciones auditivas vinculadas a procesamiento de orden superior. En la Tabla 2, se resumen los hallazgos de los AMLR en procesamiento auditivo.

\section{Potenciales evocados auditivos de laten- cia tardía (PEALT)}

Los potenciales evocados de latencia tardía o ALLR (auditory late latency response) fueron la primera respuesta eléctrica auditiva obtenida del sistema nervioso central a través de técnicas de electroencefalografía en el año $1939^{34}$. Estos están constituidos por un complejo bifásico de cuatro ondas, dos positivas y dos negativas que aparecen normalmente entre los primeros 50 a $350 \mathrm{~ms}^{35}$ (Figura 3). Son potenciales de origen cortical que, a diferencia de otras respuestas de corteza auditiva, son componentes obligatorios de naturaleza exógena, determinados por las características físicas del estímulo utilizado para evocarlos y que no requieren de la atención ni de alguna tarea en particular por parte del sujeto evaluado. Sin embargo, se han reportado algunas influencias del estado de vigilia en su obtención, por lo que sería necesario que el sujeto se mantenga despierto y alerta durante la evaluación ${ }^{35}$. Dentro de sus principales aplicaciones clínicas en la actualidad, destaca la estimación de los umbrales auditivos conductuales, evaluación y seguimiento de procesos que involucran un entrenamiento auditivo, evaluación de la sincronización neural en usuarios de implante coclear y evaluación del éxito de la adaptación de audífonos ${ }^{36}$. A pesar de que también se menciona su utilidad en la evaluación del procesamiento auditivo propiamente tal, los reportes en relación con su 
Tabla 2. Resumen de las principales investigaciones y sus hallazgos sobre la utilidad de los AMLR en el estudio del procesamiento auditivo y sus alteraciones

\begin{tabular}{|c|c|c|c|c|c|c|}
\hline Estudio & Año & Alteración & $\mathrm{n}$ & Edades & Descripción & Hallazgos \\
\hline $\begin{array}{l}\text { Schochat y } \\
\text { cols. }{ }^{22}\end{array}$ & 2004 & $\begin{array}{l}\text { TPA y } \\
\text { lesiones del } \\
\text { SNAC }\end{array}$ & 27 & 15 a 55 & $\begin{array}{c}\text { Determinar } \\
\text { sensibilidad y } \\
\text { especificidad para TPA }\end{array}$ & $\begin{array}{l}\text { - Efecto electrodo con } \\
\text { sensibilidad de } 50 \% \text { para TPA y } \\
55,6 \% \text { para lesiones del SNAC, } \\
\text { con especificidad de } 55,6 \% \text {. } \\
\text { - Efecto oído con sensibilidad } \\
\text { de } 76,5 \% \text { para TPA y } 70 \% \\
\text { para lesiones del SNAC, con } \\
\text { especificidad de } 55,6 \%\end{array}$ \\
\hline $\begin{array}{l}\text { Purdy y } \\
\text { cols. }^{28}\end{array}$ & 2002 & TEA & 10 & 7 a 11 & $\begin{array}{c}\text { Comparar con sujetos } \\
\text { normales y determinar } \\
\text { concordancia con } \\
\text { pruebas conductuales } \\
\text { SSW y CW }\end{array}$ & $\begin{array}{l}\text { - Componente Na con latencia } \\
\text { aumentada. } \\
\text { - Componente Nb con amplitud } \\
\text { reducida } \\
\text { - Concordancia con SSW y } \\
\text { reportes subjetivo de profesores }\end{array}$ \\
\hline $\begin{array}{l}\text { Frizzio y } \\
\text { cols. }^{29}\end{array}$ & 2012 & TEA & 25 & 8 a 14 & $\begin{array}{c}\text { Comparar con sujetos } \\
\text { normales }\end{array}$ & $\begin{array}{l}\text { - Componentes Pa y } \mathrm{Nb} \text { con } \\
\text { latencias aumentadas } \\
\text { - Mayores latencias en hemisferio } \\
\text { izquierdo }\end{array}$ \\
\hline $\begin{array}{l}\text { Schochat y } \\
\text { cols. }{ }^{31}\end{array}$ & 2010 & TPA & 30 & 8 a 14 & $\begin{array}{c}\text { Comparar pre y } \\
\text { posentrenamiento } \\
\text { auditivo y } \\
\text { concordancia con PSI, } \\
\text { DD, SIN y SSW }\end{array}$ & $\begin{array}{l}\text { - Componentes Na-Pa con } \\
\text { amplitudes reducidas pre } \\
\text { entrenamiento, y aumento de } \\
\text { estas post entrenamiento en } \\
\text { grupo con TPA } \\
\text { - Concordancia con PSI, DD, SIN y } \\
\text { SSW }\end{array}$ \\
\hline $\begin{array}{l}\text { Mattsson y } \\
\text { cols. }^{32}\end{array}$ & 2019 & $\begin{array}{l}\text { TPA y quejas } \\
\text { afines }\end{array}$ & 28 & 8 a 14 & $\begin{array}{l}\text { Comparar con sujetos } \\
\text { normales y determinar } \\
\text { correlación con } \\
\text { pruebas conductuales } \\
\text { FW, DD, CW, PPS, } \\
\text { DPS, GIN y BMLD }\end{array}$ & $\begin{array}{l}\text { - Componente Pa con latencias } \\
\text { aumentadas } \\
\text { - Mayores latencia al estimular en } \\
\text { oído izquierdo }\end{array}$ \\
\hline $\begin{array}{l}\text { Romero y } \\
\text { cols. }^{33}\end{array}$ & 2015 & TEA & 25 & 8 a 14 & $\begin{array}{l}\text { Correlacionar con } \\
\text { prueba de conciencia } \\
\text { fonológica }\end{array}$ & $\begin{array}{l}\text { - Latencias de componentes } \\
\mathrm{Na} \text {, Pa y Nb correlacionadas } \\
\text { negativamente con rendimiento } \\
\text { en conciencia fonológica } \\
\text { - Amplitudes de complejo } \mathrm{Na}-\mathrm{Pa} \\
\text { correlacionado positivamente } \\
\text { con rendimiento en conciencia } \\
\text { fonológica }\end{array}$ \\
\hline
\end{tabular}

TEA: trastorno específico de aprendizaje; TPA: trastorno del procesamiento auditivo; SNAC: sistema nervioso auditivo central; SSW: prueba de palabas espondaicas; CW: prueba de palabras competitivas; PSI: índice de habla pediátrico; DD: dígitos dicóticos; SIN: habla en ruido; FW: palabras filtradas; PPS: prueba de patrones de frecuencia; DPS: prueba de patrones de duración; GIN: prueba de gaps en ruido; BMLD: prueba de interacción binaural. 


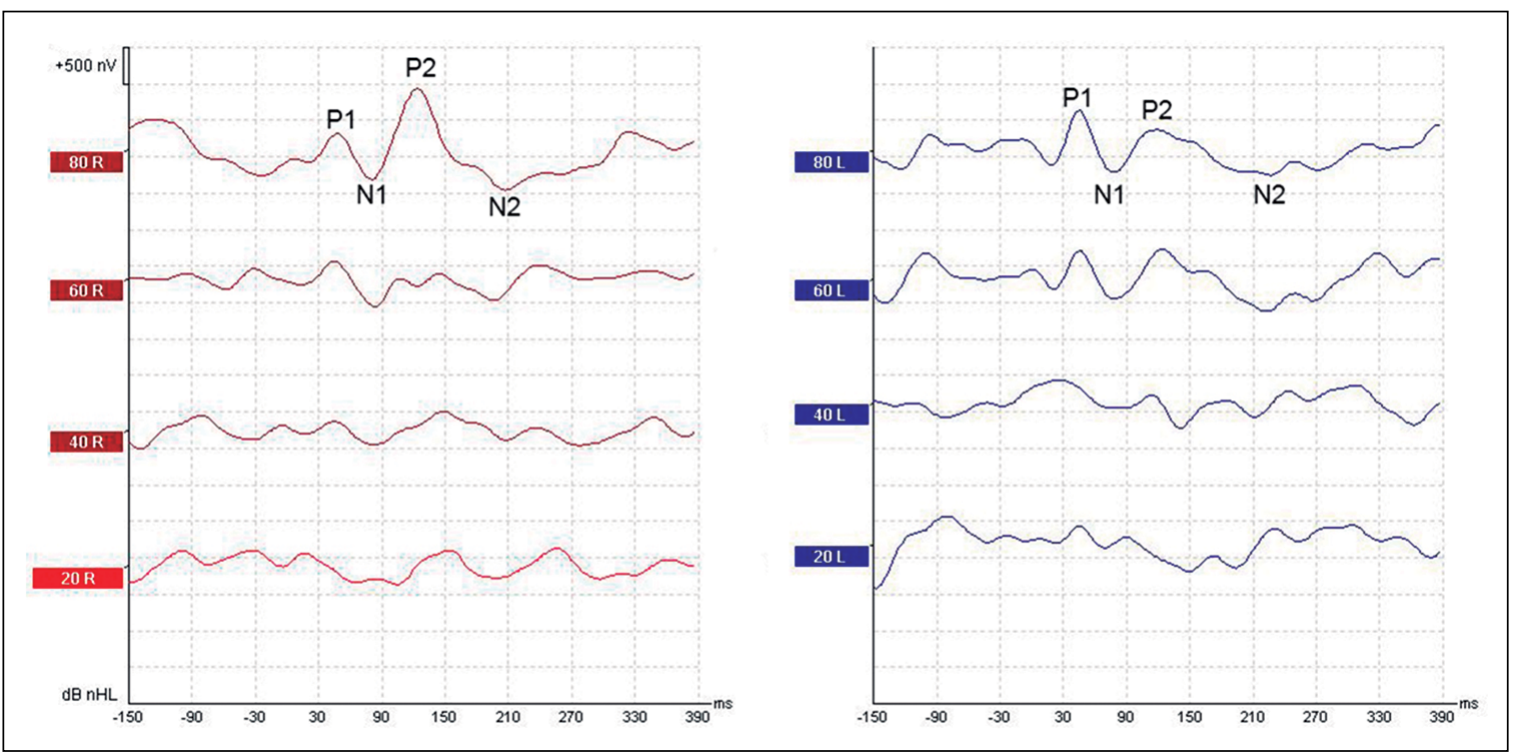

Figura 3. Potencial evocado auditivo de latencia tardía obtenido a través de un estímulo clic. Se observan los componentes P1, N1, P2 y N2.

utilidad en casos de TPA son mucho menores que sus otras aplicaciones.

En $1962^{37}$, se etiquetaron por primera vez los cuatro componentes de los ALLR, utilizando, al igual que los MLR, las letras "P" y "N" para designar a aquellos picos positivos $\mathrm{y}$ negativos respectivamente, agregando números arábigos para diferenciar las ondas según su orden de aparición. Por consiguiente, las ondas de los ALLR quedaron designados de la siguiente manera: P1 con una latencia de aparición alrededor de los $50 \mathrm{~ms}, \mathrm{~N} 1$ con una latencia en el rango de 90 y $150 \mathrm{~ms}$, P2 con latencias entre 160 y $200 \mathrm{~ms}$, y N2 que puede llegar a tener su aparición entre los 300 y 350 $\mathrm{ms}^{35}$. Actualmente, los centros generadores a los cuales se les atribuye el origen de estas respuestas van desde la corteza auditiva primaria para los componentes $\mathrm{P} 1, \mathrm{~N} 1$ y $\mathrm{P} 2$, así como aportes talámicos más específicos para P1 y del área de asociación ${ }^{38}$ y del giro de Heschl tanto para P1 como N1 $1^{39}$. P2, además, parece tener una contribución de la corteza auditiva secundaria ${ }^{39}$. Se ha documentado que la generación del componente N2 es una contribución entre el lóbulo frontal, el sistema límbico y algunas estructuras subcorticales difusas $^{40}$.

Los ALLR cuentan con un gran volumen de bibliografía, sin embargo, los estudios en relación con dificultades de procesamiento auditivo y poblaciones similares son escasos. $\mathrm{Al}$ respecto, se ha documentado que niños con TEA presentan una reducción significativa de la amplitud del componente N1 y, como consecuencia, una disminución significativa de la amplitud del complejo N1-P2 ${ }^{28}$, además de una reducción significativa en la latencia del componente P1. En sujetos sin TEA, pero con antecedentes de dificultades en la lectoescritura, se ha evidenciado que el componente N2 tiene una amplitud significativamente reducida en relación con pares con desarrollo normal ${ }^{42}$. En niños con TPA, se han medido los ALLR en condiciones normales y en presencia de ruido de fondo. En condiciones sin ruido de fondo, se ha evidenciado que los menores con TPA también presentarían un componente N2 alterado con una amplitud ${ }^{41}$ y latencia ${ }^{42}$ reducidas. En presencia de ruido de fondo, tanto sujetos con desarrollo normal como con TPA presentan una disminución significativa en la amplitud y un aumento de latencia del componente N2, además de una reducción de la amplitud del componente P1, hallazgo que también sería común para condiciones sin ruido ${ }^{41}$. A pesar de que los ALLR se tienen abundante documentación en relación con su utilidad en 


\begin{tabular}{|c|c|c|c|c|c|c|}
\hline Estudio & Año & Alteración & $\mathbf{n}$ & Edades & Descripción & Hallazgos \\
\hline $\begin{array}{l}\text { Purdy y } \\
\text { cols. }^{28}\end{array}$ & 2002 & TEA & 10 & 7 a 11 & $\begin{array}{l}\text { Comparar con sujetos } \\
\text { normales y determinar } \\
\text { concordancia con } \\
\text { prueba conductual } \\
\text { SSW y CW }\end{array}$ & $\begin{array}{l}\text { - Amplitud del componente N1 y } \\
\text { complejo N1-P2 reducida } \\
\text { - Latencia del componente P1 con } \\
\text { latencia reducida }\end{array}$ \\
\hline $\begin{array}{l}\text { Barker y } \\
\text { cols. }^{41}\end{array}$ & 2017 & $\begin{array}{l}\text { Dificultades de } \\
\text { lectoescritura } \\
\text { y TPA + } \\
\text { dificultades de } \\
\text { lectoescritura }\end{array}$ & 17 & 9 a 11 & $\begin{array}{c}\text { Comparar con sujetos } \\
\text { normales }\end{array}$ & $\begin{array}{l}\text { - Amplitud del componente } \\
\text { N2 reducida en grupo con } \\
\text { dificultades de lectoescritura } \\
\text { - Latencia del componente N2 } \\
\text { reducida en grupo de TPA + } \\
\text { dificultades de lectoescritura }\end{array}$ \\
\hline $\begin{array}{l}\text { Sharma y } \\
\text { cols. }^{42}\end{array}$ & 2014 & TPA & 55 & 7 a 13 & $\begin{array}{c}\text { Comparar con sujetos } \\
\text { normales en ruido y } \\
\text { silencio, y comparar } \\
\text { test-retest }\end{array}$ & $\begin{array}{l}\text { - Amplitud del componente P1 } \\
\text { reducida en presencia de ruido } \\
\text { - Amplitud del componente N2 } \\
\text { reducida sin ruido } \\
\text { - Componente N2 con poca } \\
\text { confiabilidad test-retest }\end{array}$ \\
\hline
\end{tabular}

TEA: trastorno específico de aprendizaje; TPA: trastorno del procesamiento auditivo; SSW: prueba de palabas espondaicas; CW: prueba de palabras competitivas.

monitoreo de intervenciones auditivas basadas en audioprótesis y en entrenamiento auditivo, no se han descrito experiencias en población con TPA y condiciones similares, y no se han logrado establecer relaciones o concordancias con pruebas conductuales de procesamiento auditivo. Estos y los demás hallazgos se resumen la en Tabla 3.

\section{Potencial de disparidad}

El potencial de disparidad o MMN (del inglés Mismatch Negativity) fue descrito por primera vez por Näätänen y colaboradores en el año $1978^{43}$. Se caracteriza por ser un componente negativo e independiente de la atención del sujeto, generada por la respuesta automática del cerebro a cualquier cambio en la estimulación auditiva que exceda un cierto límite y que se corresponde aproximadamente con el umbral de discriminación conductual ${ }^{44}$. Este potencial no solo refleja la discriminación auditiva preatencional, sino que además refleja la activación de la memoria ecoica a través de la utilización del paradigma oddball de estimulación, en el cual una secuencia de estimulación, un estímulo estándar denominado estímulo frecuente, es remplazado a veces por un estímulo discrepante, llamado también estímulo infrecuente ${ }^{45}$. El primero de ellos, se presenta generalmente en una proporción de un $80 \%-90 \%$ de la secuencia de estimulación, mientras que el estímulo infrecuente se presenta alrededor del 10\%$20 \%$ de las veces ${ }^{45}$. Ambos estímulos, tanto el estándar como el discrepante, producen las respuestas corticales clásicas ( $\mathrm{P} 1, \mathrm{~N} 1$ y $\mathrm{P} 2)$, pero sólo el estímulo discrepante produce un desplazamiento negativo, por lo que para obtener el MMN, se deben restar los potenciales promediados del estímulo estándar y del discrepante ${ }^{46}$.

La aparición del componente del MMN se da alrededor de los 100 a 250 milisegundos desde el inicio del estímulo discrepante ${ }^{35}$ (Figura 4). Mientras mayor sea la diferencia entre el estímulo frecuente y el discrepante, mayor será la amplitud del MMN y menor será su latencia de aparición ${ }^{47}$, sin embargo, mientras mayores sean las diferencias, más 


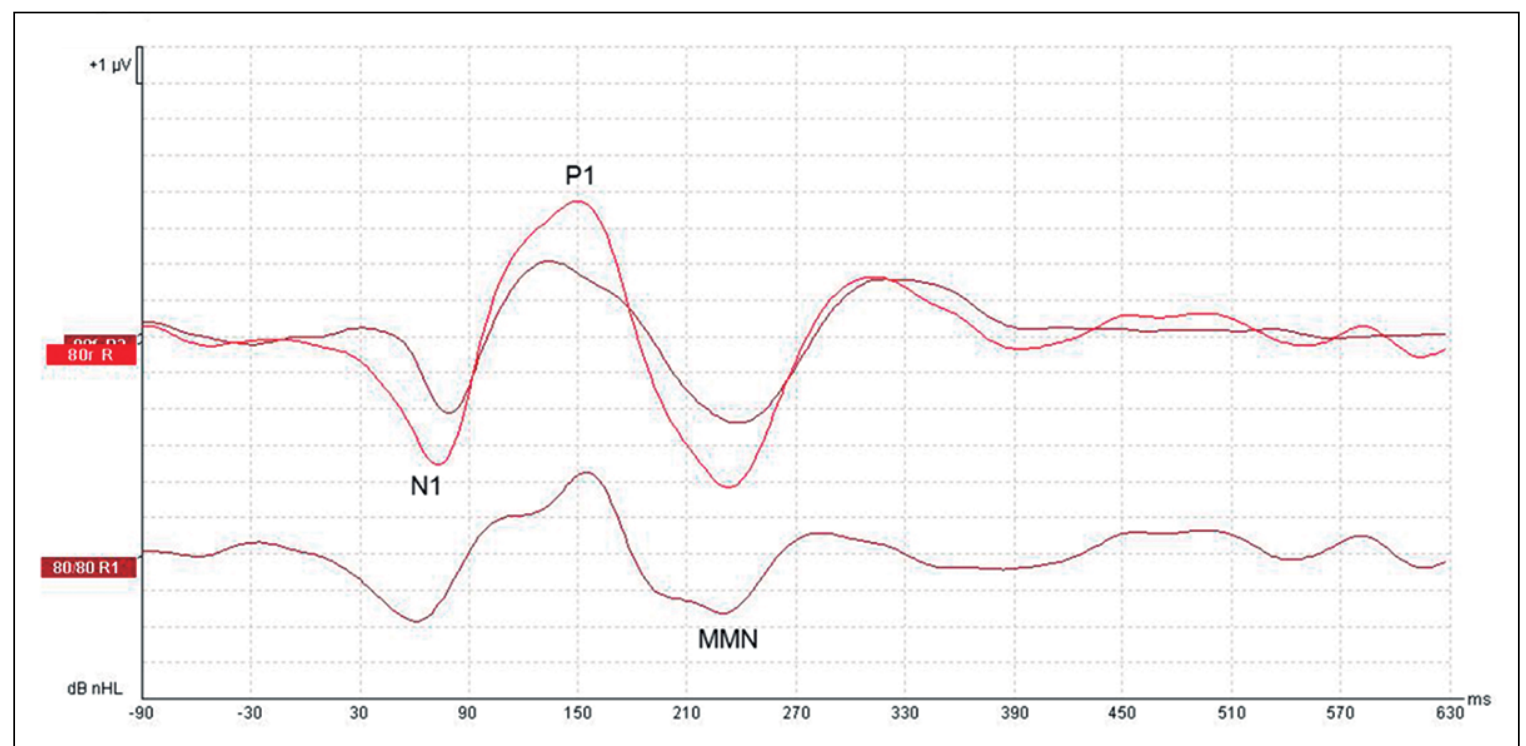

Figura 4. Potencial de disparidad identificado en la curva diferencial (abajo) utilizando un estímulo frecuente de $1000 \mathrm{~Hz}$ y uno infrecuente de $2000 \mathrm{~Hz}$. Se observan los componentes N1 y P1 en las curvas del estímulo frecuente e infrecuente (arriba).

difícil es para el individuo evaluado ignorar el estímulo, por lo que otros componentes sensoriales de respuestas evocadas auditivas podrían superponerse al MMN y dificultar su identificación ${ }^{48}$. En la actualidad, se cree que los centros generadores de este componente se encuentran en la parte superior del lóbulo temporal, involucrando tanto la corteza auditiva primaria y secundaria con algunos aportes de estructuras subcorticales y del lóbulo frontal ${ }^{49}$. Se ha documentado que dentro de las principales aplicaciones clínicas del MMN se encuentra la evaluación del procesamiento auditivo, aunque también se ha descrito su utilidad para la evaluación de alteraciones lingüísticas, autismo, implante coclear y para el pronóstico de pacientes con traumatismos craneoencefálicos en coma ${ }^{46}$.

Uno de los primeros estudios en que utilizó el MMN como método de evaluación del procesamiento auditivo fue un reporte de caso de un adulto con TPA producto de una hidrocefalia subclínica, evidenciado por dificultades severas de habla en ruido ${ }^{50}$. Las amplitudes del MMN se encontraron aumentadas al comparar con valores de referencia debido a compensaciones neuronales a nivel cortical. En menores con TPA, la evidencia ha resultado ser contradictoria. Por un lado, no se han evidenciado diferencias significativas de latencia ni amplitud entre niños con TPA y niños con desarrollo normal, justificándose en que no todos los sujetos con dificultades de procesamiento auditivo tendrían dificultades específicas de discriminación ${ }^{52}$. Por otro lado, se ha reportado que los niños con TPA tendrían un MMN con latencia significativamente aumentada en comparación con niños de audición normal ${ }^{53}$. En un estudio realizado en niños con dificultades de lectoescritura, no se logró evidenciar ninguna diferencia al compararse con pares normales utilizando estímulos frecuenciales ${ }^{51}$. Sin embargo, al utilizar estímulos verbales, se logró observar que las amplitudes se encontraron considerablemente reducidas en comparación con los controles. En menores con TEL, los hallazgos indican que tanto la amplitud como la latencia del MMN se encontrarían alteradas en comparación con sujetos con desarrollo normal, estando la amplitud reducida y la latencia aumentada ${ }^{53}$. $\mathrm{Al}$ igual que los ALLR, no se han reportado experiencias con entrenamiento auditivo ni correlaciones con pruebas conductuales de procesamiento auditivo. Se puede observar este y otros hallazgos en la Tabla 4. 
Tabla 4. Resumen de las principales investigaciones y sus hallazgos sobre la utilidad del MMN en el estudio del procesamiento auditivo y sus alteraciones

\begin{tabular}{|c|c|c|c|c|c|c|}
\hline Estudio & Año & Alteración & $\mathbf{n}$ & Edades & Descripción & Hallazgos \\
\hline $\begin{array}{l}\text { Kraus y } \\
\text { cols. }^{50}\end{array}$ & 1993 & TPA & 1 & 18 & $\begin{array}{c}\text { Estudio de caso de } \\
\text { hidrocefalia subclínica }\end{array}$ & $\begin{array}{l}\text { - Amplitudes del MMN } \\
\text { aumentadas }\end{array}$ \\
\hline $\begin{array}{l}\text { Roggia y } \\
\text { Colares }^{51}\end{array}$ & 2008 & TPA & 8 & 9 a14 & $\begin{array}{c}\text { Comprar con sujetos } \\
\text { normales }\end{array}$ & $\begin{array}{l}\text { - Sin diferencias significativas } \\
\text { en ningún parámetro del } \\
\text { MMN evocado con estímulos } \\
\text { frecuenciales }\end{array}$ \\
\hline $\begin{array}{l}\text { Rocha y } \\
\text { cols. }^{52}\end{array}$ & 2015 & TPA y TEL & 50 & 6 a 12 & $\begin{array}{c}\text { Comparar con sujetos } \\
\text { normales }\end{array}$ & $\begin{array}{l}\text { - MMN no identificable en todos } \\
\text { los casos de TPA y TEL } \\
\text { - Latencias aumentada en sujetos } \\
\text { con TPA y TEL } \\
\text { - Amplitudes reducidas en sujetos } \\
\text { con TEL }\end{array}$ \\
\hline $\begin{array}{l}\text { Sharma y } \\
\text { cols. }^{53}\end{array}$ & 2006 & $\begin{array}{l}\text { Dificultades de } \\
\text { lectoescritura }\end{array}$ & 37 & 8 a 12 & $\begin{array}{l}\text { Comparación de } \\
\text { sujetos normales, } \\
\text { con dificultades } \\
\text { de lectoescritura } \\
\text { presentes y superadas }\end{array}$ & $\begin{array}{l}\text { - Sin diferencias significativas } \\
\text { en ningún parámetro del } \\
\text { MMN evocado con estímulos } \\
\text { frecuenciales } \\
\text { - Amplitud de MMN evocado } \\
\text { por estímulos verbales con } \\
\text { amplitud reducida en grupo con } \\
\text { dificultades de lectoescritura }\end{array}$ \\
\hline
\end{tabular}

\section{Potencial P300}

El componente P3 o P300 fue descrito por primera vez en $1965^{54}$, y corresponde a una onda positiva que aparece entre los 250 y 350 $\mathrm{ms}$, pudiendo extenderse hasta los 500-600 ms tras la estimulación auditiva (Figura 5). Es obtenida mediante el paradigma oddball de estimulación, pero a diferencia del MMN, se evidencia cuando el sujeto discrimina conscientemente el estímulo infrecuente del frecuente ${ }^{35}$. Debido a que se requiere de la atención y de la participación consciente del sujeto, por ejemplo, contando la cantidad de estímulos infrecuentes mentalmente, pulsando un botón o realizando algún tipo de actividad manual, en ocasiones este potencial recibe el nombre de potencial cognitivo. Por este mismo motivo, es considerado un potencial endógeno, es decir, depende menos de las características del estímulo, pero dependen mucho del contexto del estímulo, como un cambio en la estimulación en curso, el estado del sujeto, la atención al estímulo y la cognición. Este potencial ha sido ampliamente utilizado para estudiar la atención auditiva en distintos tipos de poblaciones y con distintas condiciones de base, como déficit atencional con hiperactividad, disfluencia, alteraciones neurológicas diversas, trastornos psiquiátricos, entre otros ${ }^{55}$ $y$, a diferencia de otros potenciales auditivos, este componente tiene sus homólogos en otras modalidades sensoriales como la visual y somatosensorial ${ }^{35}$.

Uno de los primeros registros de la utilización del P300 para la evaluación del procesamiento auditivo, fue en el año $1990^{56}$. Se estudió un grupo de niños con sospecha de TPA que, al ser comparados con menores con desarrollo normal, evidenciaron latencias aumentadas y amplitudes reducidas en los componentes N1, P2 y P300. Puntualmente, los hallazgos en relación con la latencia y amplitud del P300 han podido ser corroborados en estudios posteriores ${ }^{33,57}$. Además, se ha descrito que los menores con TPA no tendrían 


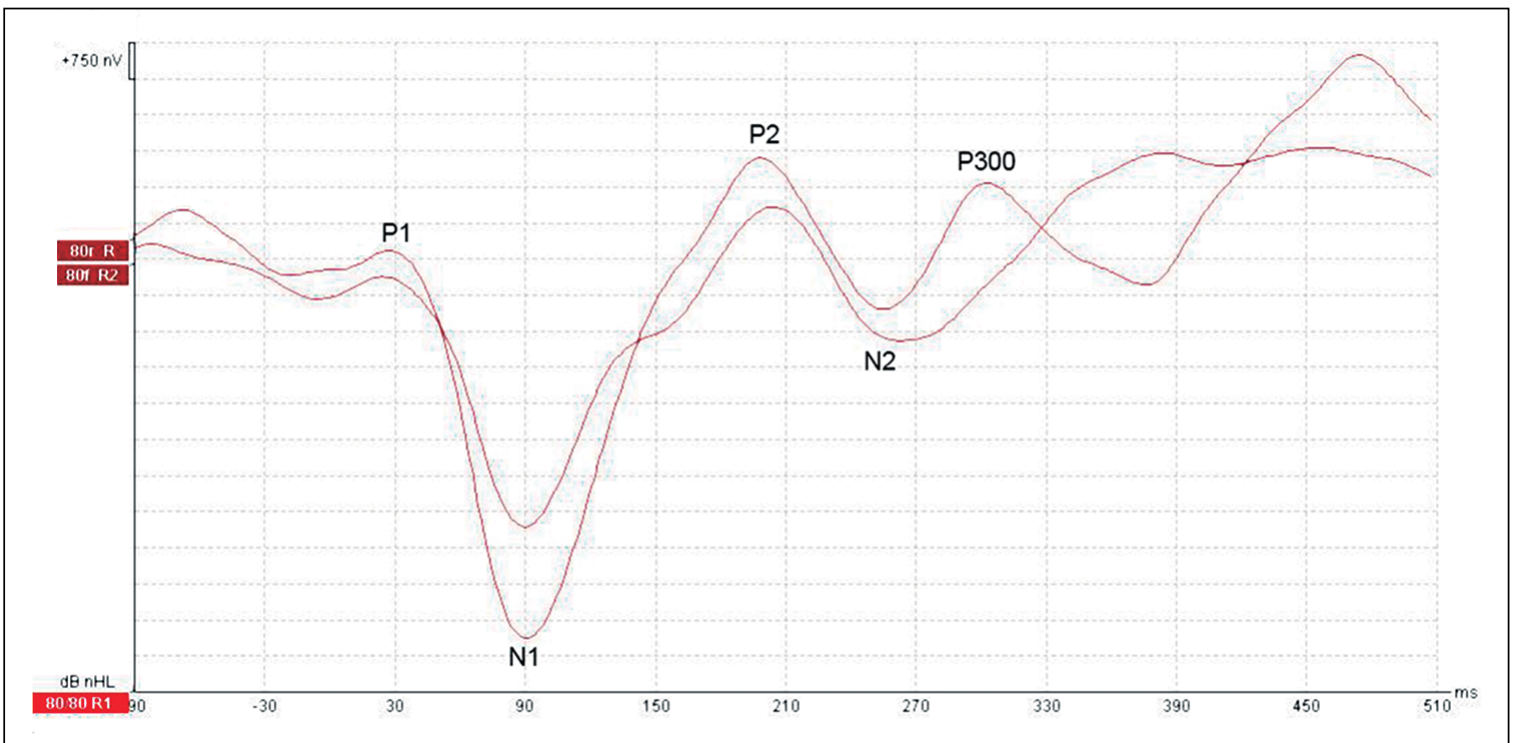

Figura 5. Componente P300 identificado utilizando un estímulo frecuente de $1000 \mathrm{~Hz}$ y uno infrecuente de $2000 \mathrm{~Hz}$. Se observan los componentes P1, N1, P2 y N2 tanto en la curva del estímulo frecuente como infrecuente.

diferencias de amplitud entre los componentes P2 y P300, mientras que en pares normales la amplitud del P300 sería significativamente mayor a la amplitud del P2 ${ }^{57}$. Los adultos con TPA mantendrían en común el hallazgo de una mayor latencia del componente P300 en comparación a pares normales, la cual sería mayor aun cuando se realiza esta medición bajo una condición de ruido competitivo ${ }^{58}$. Esta diferencia de latencias entre condiciones de silencio y de ruido competitivo no se presentaría en sujetos adultos con audición normal.

En la literatura, destaca el reporte de Jir$\mathrm{sa}^{57}$ en relación con la utilización del P300 como marcador neurobiológico del éxito de terapias auditivas en población con TPA ${ }^{57}$. En dicha investigación, se estudiaron tres grupos de niños: un grupo de niños normales como grupo control y dos grupos de niños con TPA, uno de los cuales no recibió ningún tipo de intervención y otro que fue sometido a un entrenamiento auditivo intensivo, en sesiones de 45 minutos dos veces por semana durante 14 semanas. Este entrenamiento incluyó ejercicios de memoria auditiva, comprensión de lenguaje, discriminación, atención e interpretación de instrucciones auditivas. En las mediciones preintervención no se evidenciaron diferencias entre los grupos con TPA, pero ambos presentaron latencias aumentadas y amplitudes reducidas del componente P300 en relación con el grupo de sujetos normales. Sin embargo, una vez finalizado el entrenamiento, se observó una reducción de las latencias y un aumento de las amplitudes en el grupo intervenido, al punto de no presentar diferencias significativas con el grupo control. Este estudio sugirió el aporte que puede entregar el P300 para documentar de manera objetiva los resultados de una intervención terapéutica en el TPA centrada en memoria auditiva, atención y procesamiento auditivo en general.

Por último, en relación con la correlación y/o concordancia de las mediciones del P300 con las pruebas conductuales de procesamiento auditivo, destacan principalmente dos estudios. En el primero de ellos, se estudió un grupo de menores que cumplían criterios diagnósticos de TPA y otro grupo que, a pesar de no cumplirlos, tenían reportes de quejas auditivas $^{33}$. En ambos grupos se evidenció una correlación positiva entre la amplitud del P300 y las pruebas de palabras competitivas (CW), prueba de patrones de frecuencia (PPS), la prueba de patrones de duración (DPS) y dígitos dicóticos. También se lograron evidenciar 
correlaciones significativas negativas entre la latencia del P300 y las pruebas de patrones de frecuencia y dígitos dicóticos. En un segundo estudio $^{57}$, si bien no se analizaron correlaciones, se evidenció una concordancia entre latencias aumentadas y amplitudes reducidas con resultados deficientes en una prueba conductual de atención selectiva auditiva (Selective Auditory Attention Competing) y en una batería de pruebas conductales de diversas habilidades auditivas (GFW). Estos y los demás hallazgos se resumen en la Tabla 5.

\section{Conclusión}

Según los artículos revisados, el principal parámetro diferenciador entre población con desarrollo normal y población con alteraciones del procesamiento auditivo es la amplitud de algunos componentes. En el caso de los cABR, los componentes que se presentan con amplitudes reducidas son el FFR, el Va y el C. En los AMLR se observan amplitudes disminuidas principalmente en el componente $\mathrm{Nb}$. Para los ALLR, los principales hallazgos están en el componente N1 que también se encuentra con una menor amplitud, aunque se ha evidenciado esta tendencia en los componentes P1 y N2. No se encontraron alteraciones de la amplitud en el MMN, sin embargo, en el P300 se evidencia el mismo hallazgo que en el resto de los exámenes. Existe cierta ambigüedad en relación con la utilidad de las latencias de aparición de los componentes, por lo que su uso como elemento diferenciador entre la normalidad auditiva y algún tipo de alteración es limitado. Existen otros indicadores de alteración como, por ejemplo, diferencias interaurales, diferencias de electrodos, ausencia de componentes, entre otros. Sin embargo, estos reportes son menos consistentes en la literatura revisada.

Otro antecedente para tener en consideración al momento de utilizar alguna técnica de evaluación electrofisiológica es su correlación con las pruebas conductuales de procesamiento auditivo disponibles para utilización clínica.

Tabla 5. Resumen de las principales investigaciones y sus hallazgos sobre la utilidad del P300 en el estudio del procesamiento auditivo y sus alteraciones

\begin{tabular}{|c|c|c|c|c|c|c|}
\hline Estudio & Año & Alteración & $\mathbf{n}$ & Edades & Descripción & Hallazgos \\
\hline $\begin{array}{l}\text { Mattsson y } \\
\text { cols. }{ }^{33}\end{array}$ & 2019 & $\begin{array}{l}\text { TPA y quejas } \\
\text { afines }\end{array}$ & 28 & 8 a 14 & $\begin{array}{l}\text { Comparar con sujetos } \\
\text { normales y determinar } \\
\text { correlación con } \\
\text { pruebas conductuales } \\
\text { FW, DD, CW, PPS, } \\
\text { DPS, GIN y BMLD. }\end{array}$ & $\begin{array}{l}\text { - Amplitudes reducidas y latencias } \\
\text { aumentadas en P300 } \\
\text { - Correlaciones positivas entre } \\
\text { P300 y DD, PPS y DPS }\end{array}$ \\
\hline $\begin{array}{l}\text { Jirsa y } \\
\text { Clontz }^{56}\end{array}$ & 1990 & TPA & 24 & 9 a 11 & $\begin{array}{c}\text { Comparar con sujetos } \\
\text { normales }\end{array}$ & $\begin{array}{l}\text { - Amplitudes reducidas y latencias } \\
\text { aumentadas para N1, P2 y P300 } \\
\text { - Concordancia con prueba } \\
\text { conductual de atención auditiva }\end{array}$ \\
\hline Jirsa $a^{57}$ & 1992 & TPA & 20 & 9 a 12 & $\begin{array}{c}\text { Comparar con } \\
\text { sujetos normales pre } \\
\text { y posentrenamiento } \\
\text { auditivo }\end{array}$ & $\begin{array}{l}\text { - Amplitudes reducidas y } \\
\text { latencias aumentadas en P300 } \\
\text { preentrenamiento y ambas } \\
\text { normales posentrenamiento }\end{array}$ \\
\hline Krishnamurti58 & 2001 & TPA & $10^{\prime}$ & Adultos & $\begin{array}{l}\text { Comparar con } \\
\text { sujetos normales con } \\
\text { estimulación binaural } \\
\text { y en presencia de } \\
\text { ruido }\end{array}$ & $\begin{array}{l}\text { - Latencias aumentadas en } \\
\text { componente P300 en ambas } \\
\text { condiciones, mayor para } \\
\text { condición con ruido }\end{array}$ \\
\hline
\end{tabular}

TPA: trastorno del procesamiento auditivo; FW: palabras filtradas; DD: dígitos dicóticos; CW: prueba de palabras competitivas; PPS: prueba de patrones de frecuencia; DPS: prueba de patrones de duración; GIN: prueba de gaps en ruido; BMLD: prueba de interacción binaural. 
A pesar de que se han podido evidenciar algunas correlaciones significativas entre ambas técnicas de evaluación, es esperable encontrar algunas diferencias debido a que, principalmente, las pruebas conductuales evalúan habilidades diversas y requieren de una correcta asignación de recursos cognitivos. Algunas de las técnicas electrofisiológicas descritas en el presente artículo no requieren de la participación del sujeto evaluado, por lo que además de constituir una herramienta de evaluación objetiva, podría ser un diferenciador entre una alteración del SNAC propiamente tal y un trastorno cognitivo o lingüístico de base. Sin embargo, desde una perspectiva ecológica, la mayoría de las habilidades auditivas actúan en conjunto de, por ejemplo, atención, memoria y lenguaje, como en el caso de la localización y lateralización sonora, desempeño auditivo con señales acústicas competitivas o con señales acústicas degradadas, entre otras. Por este motivo, no sería esperable encontrar correlaciones directas entre ambos tipos de evaluaciones. Actualmente sigue existiendo poca claridad al respecto y, más aún, los hallazgos contradictorios entre ambas técnicas podrían dificultar la interpretación de una batería exhaustiva y podría llevar a cometer errores diagnósticos.

A pesar de que existen numerosos hallazgos sobre diferencias cuantitativas y cualitativas en las técnicas electrofisiológicas entre menores con TPA y menores con desarrollo normal, la gran variabilidad de estos hallazgos impide establecer un perfil de alteración claro para establecer la presencia o ausencia de este trastorno. Esto último, sumado a la ausencia de un protocolo estandarizado, no ha permitido establecer criterios diagnósticos útiles para la práctica clínica habitual. Por otro lado, la utilidad de estas técnicas como marcador objetivo de éxito terapéutico en intervenciones basadas en entrenamiento auditivo podría tener una utilidad clínica más factible considerando la relativamente baja variabilidad intrasujeto de estos procedimientos. Bastaría con establecer perfiles basales preintervención y realizar mediciones postintervención utilizando los mismos protocolos para realizar las comparaciones. Sin embargo, a pesar de la objetividad que entregaría la electrofisiología, esta no consideraría aspectos funcionales del procesamiento auditivo que, independiente de la actividad neurobiológica subyacente, serían un indicador con mayor validez ecológica para establecer el éxito terapéutico. Nuevamente, las pruebas conductuales de procesamiento auditivo tienen ventaja en este aspecto. Por este motivo, la utilidad de las técnicas electrofisiológicas en estos términos estaría limitada a poblaciones en las cuales no es posible realizar un adecuado condicionamiento para la batería de evaluación conductual.

Otra limitante para la utilización de este tipo de técnica, al menos a nivel nacional, es la ausencia de valores normativos, lo que impediría incluso establecer normalidad o alteración cuando los componentes de los trazados puedan ser identificados. A nivel nacional, no existen reportes de la utilización de electrofisiología para la evaluación del procesamiento auditivo. A pesar de que la ASHA, la AAA y algunos autores recomiendan su utilización, las limitantes identificadas en la presente revisión concuerdan con lo expuesto por la BSA, y parecen indicar que la evidencia es aún insuficiente para justificar este tipo de mediciones en el diagnóstico clínico del TPA y otros trastornos relacionados, así como en el monitoreo de las intervenciones basadas en entrenamiento auditivo. Si bien estas herramientas podrían ser de utilidad clínica para establecer la integridad de la vía auditiva más allá del alcance de los clásicos PEATC, se recomienda condicionar su utilización clínica hasta contar por lo menos con valores de referencia bien establecidos y protocolos de evaluación estandarizados, al menos para la evaluación del procesamiento auditivo y prefiriendo por sobre estas técnicas la batería de pruebas conductuales clínicamente disponibles.

\section{Bibliografía}

1. American Speech-Language-Hearing Association. (Central) Auditory Processing Disorders [Technical Report] [Internet]. 2005. p. 1-20. Disponible en: www.asha.org/policy

2. British Society of Audiology. Position Statement and Practice Guidance Auditory Processing Disorder (APD). 2018. Disponible en: www.thebsa.org

3. Jerger J, Musiek F. Report of the Consensus Conference on the Diagnosis of Auditory Processing Disorders in School-Aged Children. J Am Acad Audiol. 2000;11(9):467-474. 
4. American Academy of Audiology. Clinical Practice Guidelines: Diagnosis, Treatment and Management of Children and Adults with Central Auditory Processing Disorder [Internet]. 2010. Disponible en: https://www.audiology.org/publications-resources/ document-library/central-auditory-processingdisorder.

5. Moore D, Rosen S, Bamiou D, Campbell N, Sirimanna T. Evolving concepts of developmental auditory processing disorder (APD): A British Society of Audiology APD Special Interest Group "white paper”. Int J Audiol. 2013;52(1):3-13.

6. Kraus N, Nicol T. Aggregate neural responses to speech sounds in the central auditory system. Speech Commun. 2003;41(1):35-47.

7. Kraus N, Skoe E. Auditory brainstem response to complex sounds: a tutorial. Ear Hear. 2010;31(3):302324.

8. Strait DL, Kraus N, Skoe E, Ashley R. Musical experience and neural efficiency - Effects of training on subcortical processing of vocal expressions of emotion. Eur J Neurosci. 2009;29(3):661-668.

9. Musacchia G, Sams M, Skoe E, Kraus N. Musicians have enhanced subcortical auditory and audiovisual processing of speech and music. Proc Natl Acad Sci U S A. 2007;104(40):15894-8.

10. Nicol T, Kraus N. Speech-sound encoding: physiological manifestations and behavioral ramifications. En: Supplements to Clinical Neurophysiology. Amsterdam: Elsevier B.V.; 2004. pp. 628-634.

11. Rocha C, Filippini R, Moreira R, Ferreira I, Schochat E. Brainstem auditory evoked potential with speech stimulus. Pró-Fono R Atual Cient. 2010;22(4):479-84.

12. Wible B, Nicol T, Kraus N. Atypical brainstem representation of onset and formant structure of speech sounds in children with language-based learning problems. Biol Psychol. 2004;67(3):299-317.

13. Filippini R, Schochat E. Brainstem evoked auditory potentials with speech stimulus in the auditory processing disorder. Braz J Otorhinolaryngol. 2009;75(3):449-455.

14. Filippini R, Befi-Lopes D, Schochat E. Efficacy of auditory training using the auditory brainstem response to complex sounds: Auditory processing disorder and specific language impairment. Folia Phoniatr Logop. 2012;64(5):217-226.

15. Sanguebuche T, Peixe B, Bruno R, Biaggio E, García M. Speech-evoked brainstem auditory responses and auditory processing skills: A correlation in adults with hearing loss. Int Arch Otorhinolaryngol. 2018;22(1):38-44.

16. Bayat A, Farhadi M, Emamdjomeh H, Saki N, Mirmomeni G, Rahim F. Effect of conductive hearing loss on central auditory function. Braz J Otorhinolaryngol. 2017;83(2):137-141.

17. Salvador K, Pereira T. Auditory processing in unilateral hearing loss: case report. J Soc Bras Fonoaudiol. 2011;23(41):381-4.

18. Neijenhuis K, Tschur H, Snik A. The Effect of Mild Hearing Impairment on Auditory Processing Tests. J Am Acad Audiol. 2004;15(1):6-16.

19. Kraus N. Clinical applications of the middle latency response. J Am Acad Audiol. 1990;1(3):130-133.

20. Harkrider A. Middle Latency Responses. En: Atcherson S, Stoody T, editores. Auditory Electrophysiology, A Clinical Guide. New York, NY: Thieme Medical Publishers; 2012. p. 213-238.

21. Geisler C, Frishkopf L, Rosenblith W. Extracranial responses to acoustic clicks in man. Science (80). 1958;128(3333):1210-1211.

22. Schochat E, Rabelo C, Loreti R. Sensitividade e especificidade do potencial de média latência. Rev Bras Otorrinolaringol. 2004;70(3):353-358.

23. Goldstein R, Rodman L. Early Components of Averaged Evoked Responses to Rapidly Repeated Auditory Stimuli. J Speech Hear Res. 1967;10(4):697705.

24. McGee T, Kraus N, Comperatore C, Nicol T. Subcortical and cortical components of the MLR generating system. Brain Res. 1991;544(2):211-220.

25. Woods D, Clayworth C, Knight R, Simpson G, Naeser $\mathrm{M}$. Generators of middle- and long-latency auditory evoked potentials: implications from studies of patients with bitemporal lesions. Electroencephalogr Clin Neurophysiol. 1987;68(2):132-148.

26. Yvert B, Fischer C, Bertrand O, Pernier J. Localization of human supratemporal auditory areas from intracerebral auditory evoked potentials using distributed source models. Neuroimage. 2005;28(1):140-153.

27. Kraus N, McGee T, Littman T, Nicol T. Reticular formation influences on primary and non-primary auditory pathways as reflected by the middle latency response. Brain Res. 1992;587(2):186-194.

28. Purdy S, Kelly A, Davies M. Auditory brainstem response, middle latency response, and late cortical evoked potentials in children with learning disabilities. J Am Acad Audiol. 2002;13(7):367-382.

29. Cañete O. Desorden del procesamiento auditivo central (DPAC). Rev Otorrinolaringol Cir Cabeza Cuello. 2006;66(3):263-273.

30. Schochat E, Musiek F, Alonso R, Ogata J. Effect of auditory training on the middle latency response in children with (central) auditory processing disorder. Braz J Med Biol Res. 2010;43(8):777-785.

31. Frizzo A, Issac M, Pontes-Fernandes A, Menezes $\mathrm{P}$, Funayama C. Auditory middle latency response in children with learning difficulties. Int Arch Otorhinolaryngol. 2012;16(3):335-340.

32. Romero A, Funayama R, Capellini S, Frizzo A. Auditory Middle Latency Response and Phonological Awareness in Students with Learning Disabilities. Int Arch Otorhinolaryngol. 2015;19(4):325-330. 
33. Mattsson T, Lind O, Follestad T, et al. Electrophysiological characteristics in children with listening difficulties, with or without auditory processing disorder. Int J Audiol. 2019;58(8):1-13.

34. Davis P. Effects of acoustic stimuli on the waking human brain. J Neuropsychol. 1939;2(6):494-499.

35. White L, Atcherson S. Cortical Event-Related Potentials. En: Atcherson S, Stoody T, editores. Auditory Electrophysiology, A Clinical Guide. New York, NY: Thieme Medical Publishers; 2012.

36. Cañete O. Potenciales evocados auditivos de corteza: Complejo P1-N1-P2 y sus aplicaciones clínicas. Rev Otorrinolaringol Cir Cabeza Cuello. 2014;74(3):266274.

37. Williams H, Tepas D, Morlock H. Evoked responses to clicks and electroencephalographic stages of sleep in man. Science. 1962;138(3541):685-686.

38. Ponton C, Eggermont J. Of Kittens and Kids: Altered Cortical Maturation following Profound Deafness and Cochlear Implant Use. Audiol Neurootol. 2002;6(6):363-380.

39. Lightfoot G. Summary of the N1-P2 Cortical Auditory Evoked Potential to Estimate the Auditory Threshold in Adults. Semin Hear. 2016;37(1):1-8.

40. Kiehl K, Laurens K, Duty T, Forster B, Liddle P. Neural sources involved in auditory target detection and novelty processing: An event-related fMRI study. Psychophysiology. 2001;38(1):133-142.

41. Sharma M, Purdy S, Kelly A. The contribution of speech-evoked cortical auditory evoked potentials to the diagnosis and measurement of intervention outcomes in children with auditory processing disorder. Semin Hear. 2014;35(1):51-64.

42. Barker M, Kuruvilla-Mathew A, Purdy S. Cortical Auditory-Evoked Potential and Behavioral Evidence for Differences in Auditory Processing between Good and Poor Readers. J Am Acad Audiol. 2017;28(6):534-545.

43. Näätänen R, Gaillard A, Mäntysalo S. Early selectiveattention effect on evoked potential reinterpreted. Acta Psychol. 1978;42(4):313-329.

44. Nätänen R, Paavilainen P, Rinne T, Alho K. The mismatch negativity (MMN) in basic research of central auditory processing: A review. Clin Neurophysiol. 2007;118(12):2544-2590.

45. Lang A, Eerola O, Korpilahti P, Holopainen I, Salo S, Aaltonen O. Practical issues in the clinical application of mismatch negativity. Ear Hear. 1995;16(1):117-129.
46. Carrasco L, Pavez E, Délano P. Potencial de Disparidad. Rev Otorrinolaringol Cir Cabeza Cuello. 2008;68(2):185-192.

47. Amenedo E, Escera C. The accuracy of sound duration representation in the human brain determines the accuracy of behavioural perception. Eur J Neurosci. 2000;12(7):2570-2574.

48. Näätänen R. The mismatch negativity: a powerful tool for cognitive neuroscience. Ear Hear. 1995;16(1):618.

49. Näätänen R. The role of attention in auditory information processing as revealed by event-related potentials and other brain measures of cognitive function. Behav Brain Sci. 1990;13(2):201-233.

50. Kraus N, McGee T, Ferre J, et al. Mismatch negativity in the neurophysiologic/behavioral evaluation of auditory processing deficits: A case study. Ear Hear. 1993;14(4):223-235.

51. Sharma M, Purdy S, Newall P, Wheldall K, Beaman $\mathrm{R}$, Dillon $\mathrm{H}$. Electrophysiological and behavioral evidence of auditory processing deficits in children with reading disorder. Clin Neurophysiol. 2006;117(5):1130-1144.

52. Roggia S, Colares N. Mismatch negativity in patients with (central) auditory processing disorders. Braz J Otorhinolaryngol. 2008;74(5):705-711.

53. Rocha C, Befi-Lopes D, Schochat E. Mismatch negativity in children with specific language impairment and auditory processing disorder. Braz J Otorhinolaryngol. 2015;81(4):408-415.

54. Sutton S, Braren M, Zubin J, John E. Evokedpotential correlates of stimulus uncertainty. Science (80). 1965;150(3700):1187-1188.

55. Soares E, Ferreira L, de Carvalho M, da Silva A. Applicability of the P300 frequency pattern test to assess auditory processing. Braz J Otorhinolaryngol. 2013;79(4):512-521.

56. Jirsa R, Clontz K. Long Latency Auditory EventRelated Potentials from Children with Auditory Processing Disorders. Ear Hear. 1990;11(3):222-232.

57. Jirsa R. The Clinical Utility of the P3 AERP in Children with Auditory Processing Disorders. J Speech Hear Res. 1992;35(4):903-912.

58. Krishnamurti S. P300 Auditory Event-Related Potentials in Binaural and Competing Noise Conditions in Adults with Central Auditory Processing Disorders. Contemp Issues Commun Sci Disord. 2001;28(1):40-47. 